Article

\title{
Optimization of a Kitting Line: A Case Study
}

\author{
Nicola Comand *, Riccardo Minto, Giovanni Boschetti, Maurizio Faccio and Giulio Rosati $\mathbb{D}$ \\ Department of Management and Engineering, University of Padova, Via Venezia 1, 35121 Padova, Italy \\ * Correspondence: nicola.comand@phd.unipd.it; Tel.: +39-049-827-6810
}

Received: 1 May 2019; Accepted: 5 August 2019; Published: 7 August 2019

\begin{abstract}
Assembly kitting lines typically deal with components that present different quantities in the final kit. Re-configurable feeders are not advisable for low quantity components since this solution leads to high inefficiency, due to the time required for retooling and for the feeding rate unbalance between the components. On the other hand a fully flexible feeder can increase the flexibility of the system at the cost of reducing the throughput. An assembly kitting problem was therefore addressed in different ways for low quantity components and high quantity components, studying the two different subsystems that compose a hybrid flexible assembly system. To optimize the re-configurable feeders, which supply the high quantity components, the opening sequence of a system composed by several hoppers was analyzed. We propose a solution which replaces the weighing device with a vision inspection system, showing its impact on the productivity of the line. A model coded into a Matlab script was developed to perform the optimization of the system and understand its behavior. Furthermore a fully flexible assembly system was developed in the laboratory in order to test the kitting of the low quantity components with the proposed subsystem. Finally the Overall Equipment Effectiveness of the line was calculated to evaluate the possible improvements obtained by the proposed solution.
\end{abstract}

Keywords: hopper; optimization; sequencing; kitting; H-FAS

\section{Introduction}

In order to compete in a global market, nowadays companies need to offer a wide range of different products. The production technology most sensitive to this evolution is assembly, because of its position at the end of production processes, where the whole product variety is present. As a consequence, companies try to use adaptive flexible assembly systems [1]. As reported by Barbazza et al. [2], the basic requirements for the winning design and management of a flexible assembly system concern the optimization of:

- unit direct production cost ( $€ /$ part), that is, the ratio of the hourly costs of the workcell and the average throughput;

- mix flexibility, that is, the ability to handle a wide variety of part types (components), and manage a wide mix of components and products;

- volume flexibility, that is, the ability to change the productivity of the system without reducing the efficiency of the system.

The basic assembly production strategies are traditionally classified as manual assembly systems, flexible (automated) assembly systems, and dedicated (automated) assembly systems [3]. According to Heilala and Voho [4], the transition from a dedicated to a flexible automated assembly system and then to a manual assembly system is due to the increase in the requirements of flexibility and number of variants. On the other hand, pure manual assembly has different drawbacks. The accuracy of the tasks performed and the activity repeatability need improvements. Ergonomic problems, as well as 
occupational injuries, could occur [5]. At last absenteeism can create production lack and low efficiency, especially in the case of balanced assembly lines [6]; for these reasons a pure manual assembly system cannot be very competitive, especially in countries with high labor cost levels. As affirmed by Edmondson and Redford [7], the driving factor behind the definition of a suitable assembly system configuration is, first of all, economy. Automation is usually a way to lower the costs of labor in Western countries and the key issue is to achieve and optimize flexibility and a suitable degree of automation in a fast changing market situation [8]. Therefore, in the last decades, a subset of flexible automated assembly systems have been developed with the aim of increasing flexibility and decreasing production costs if compared with the traditional manual and automated assembly systems.

The traditional flexible assembly system (FAS) is typically composed of one programmable manipulator, which picks the parts from predefined positions, places them on the assembly station and perform the assembly task and one or more re-configurable feeding devices, typically vibratory bowl feeders, for each component needed to complete the production process. An evolution of the concept of the flexible assembly systems is presented in Reference [9], fully FAS (F-FAS). The F-FAS is a single-station robotized assembly system, able to perform mixed model assembly, where the unique feeding device is a fully flexible feeder, ensuring a higher level of flexibility than the traditional automated FAS. The feeder system, since it is composed by a bulk, a vibratory plane and a camera, can feed several different components without the need for reconfiguration. As far as this solution presents high levels of flexibility, it has a lower throughput than a traditional FAS, since image processing for shape recognition is time consuming and the feeding process is stochastic.

In order to bridge the gap between the presented solutions, the hybrid FAS (H-FAS) has been developed [10]. The H-FAS consists of one programmable manipulator, one flexible feeder and one or more re-configurable feeder devices like the vibratory bowl feeder. Lowering the number of parts deployed by the fully flexible feeder leads to a reduction in the complexity of the system, thus increasing the throughput. It should be noted that the H-FAS is able to reach performances, in terms of unit direct production costs, mix flexibility and volume flexibility, higher than those of the other systems presented. The advantages of using this type of solution can be noticed when the feeding process is optimized for the different components, as a function of the characteristics of each one.

The proposed work arises from a case study, which aims to improve the efficiency of an assembly kitting line. The assembly kitting process is widely used for different purposes, for example, sales kits or production kit. A typical application of the studied assembly kitting line is the production of sales kits of connectors, such as screws and bolts, ready to use in the product assembly process.

Such a process is performed by means of a traditional FAS. The production line taken as a reference is indeed composed by 8 bowl feeders, which through a conveyor belt and three hoppers delivers the components onto the inferior belt, which moves towards the packing machine. The line is depicted in Figure 1.

A literature review analysis has led to a limited number of studies on increasing the efficiency of an assembly kitting line. Bevilacqua et al., in Reference [11], present a case study in the pharmaceutical industry, where they applied new procedures based on the lean production approach; moreover, they evaluated the increase in efficiency based on the Overall Efficiency Equipment (OEE, [12]) value, an approach suitable also for our study. In Reference [13] the authors described the design process for an automated assembly line; it should be noted that the authors have carried out a redesign of the deployed components, a solution which could not be applied in our case study.

In order to increase the efficiency in our case study, we observed that the deployment of low quantity components by means of the bowl feeders leads to high inefficiency, due to the time required for retooling and for the feeding rate unbalance between the components. For such reasons this paper investigates the possibility of applying an H-FAS model on the considered process, since we believe that for its characteristics it is more suitable than traditional ones. The aim of this work is therefore to propose an alternative solution to the kitting line layout taken as reference from the case study. The study will be divided into two problems: the first will consider the feeding of the low quantity 
components and will be solved with the development of a flexible robotic workcell, the second will deal with the feeding of the high quantity components and will lead to the improvement of the original feeding system.

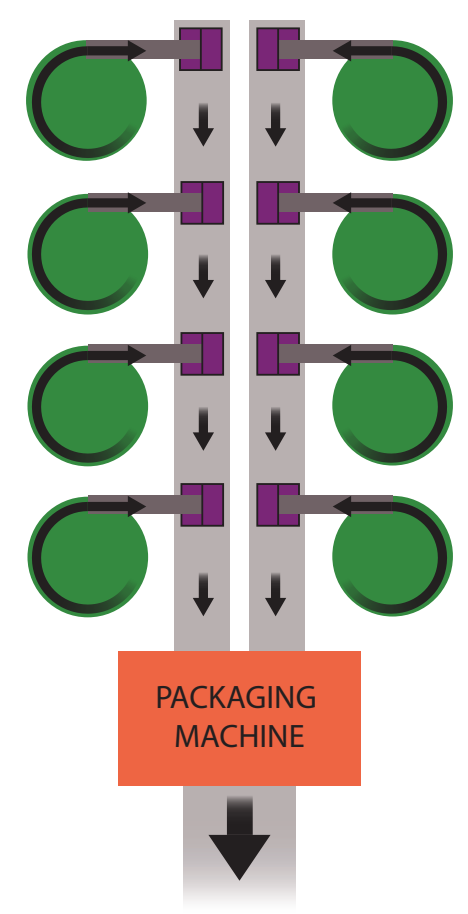

Figure 1. Line layout.

With regard to the feeding system, a focus on the hoppers cascade system was considered advantageous in the proposed case study. No one, to the authors' knowledge, has studied the impact of a specific configuration of a hoppers cascade system and its parameters (e.g., opening/closing time, output feed-rate, etc.) on its performance. Similar studies [14] have focused on continuous processing with the need for control flow rates of materials and whose models were not suitable for the presented case study.

The aim of the study will therefore be:

- $\quad$ reconfiguration of a kitting line with H-FAS systems, applied to kitting without special assembly requirements;

- modeling of the hoppers of the bowl feeders of the H-FAS system;

- demonstration of the improvement in line performance by removing some of the traditional feeders;

The novelty of the presented work, besides suggesting two different improvements for an assembly kitting line, is the study of the behavior of a hoppers cascade system based on its configuration. In this way we can estimate the throughput of a feeding system and evaluate more convenient configurations. Moreover the proposed solution was applied in a case study, validating the proposed results. The presented work is organized as follows: Section 2 presents the systems used to improve the current assembly kitting line, divided in Section 2.1 for the robotics F-FAS and Section 2.2 for the hoppers cascade system. Section 4 shows the impact of the proposed solutions and Section 3 presents results provided by the application of the model to the case study. Lastly, Section 5 concludes the work. 


\section{Materials and Methods}

This section describes the systems used to address the low and the high quantity component feeding problem. To solve the former a flexible robotic workcell was developed and experimental tests were performed to find the mean time for a pick and place operation. This is described in detail in Section 2.1. To solve the high quantity components, three configurations were modeled and by mean of simulations was evaluated the impact of some parameters on the system performance. The system analyzed is described in Section 2.2 and the sensitivity analysis of the model is presented in Section 2.2.1. The simulation is treated in particular in Section 2.2.2 and the vision inspection system in Section 2.2.3.

\subsection{Flexible Robotic Workcell}

An experimental setup is needed to evaluate the effectiveness of the usage of the adopted devices, such as flexible feeders and vertical packaging machines. A F-FAS prototype was therefore developed at the Industrial Robotics Laboratories of the University of Padua, where a robot carried out a pick and place job. The tested workcell is composed of the following devices, as represented in Figure 2:

- $\quad$ the manipulator, an Adept Viper 650 anthropomorphic arm with a $650 \mathrm{~mm}$ reach; the device is equipped with a Schunk MPG 25 pneumatic gripper with two parallel jaws.

- $\quad$ The controller Adept CX controller supported by PC-based applications for image processing (Halcon) and work cycle scheduling (Matlab); the two subsystems communicate through a TCP-IP connection.

- $\quad$ The feeding system, a Flex Factory Anyfeed SX240; this commercial flexible feeder presents a back-lighted vibratory plane where the parts from the vibratory bulk are distributed through controlled shakes.

- $\quad$ The vision system, consisting of ab AVT Pike F-505 monochrome $2452 \times 2054$ FireWire 5 Megapixel camera; the focal axis of the camera is orthogonal to the working plane and with a $35 \mathrm{~mm}$ focal length lens.

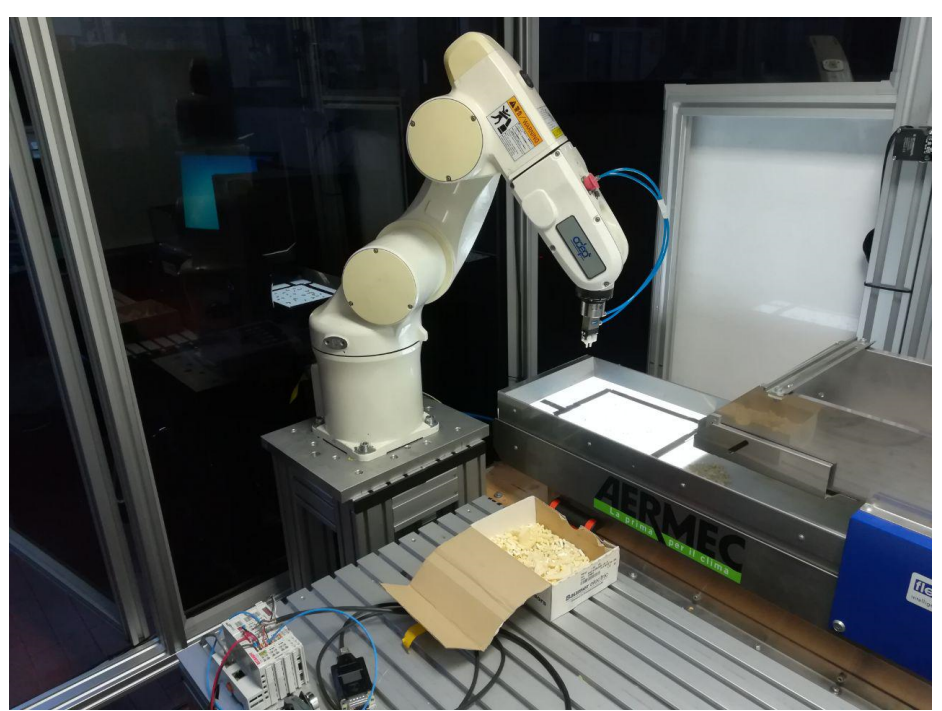

Figure 2. Prototype workcell used during the tests.

The robot picks parts of four different shapes, as represented in Figure 3, from the flexible feeder; the picking area, represented by the $170 \times 220 \mathrm{~mm}$ area enclosed within a black boundary in Figure 2, was designed in order to be the largest area where the robot could reach any point. The center of symmetry of the area is therefore placed with $\mathrm{X}, \mathrm{Y}, \mathrm{Z}$ coordinates from the robot base equal to respectively $400 \mathrm{~mm}, 365 \mathrm{~mm}, 18 \mathrm{~mm}$. 


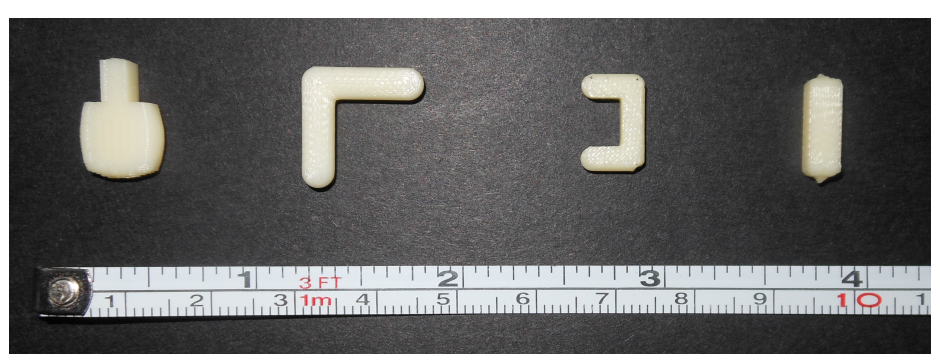

Figure 3. Parts picked by the robot in the experimental workcell.

The parts on the vibratory plane are identified by the shape matching method provided by the Halcon software [15]. The method allows the identification of an object from the image by recognizing the gray-scale level of the reference photo, with the score parameter, which is set equal to 0.7 , representing how faithful is the image to the recognized part. The software is also provided of a function that identifies the pickup location on the part which differs for each shape and it is defined in order to avoid to lose grip.

After the image processing was carried out, the system initiates the pick and place job, which consists of the following tasks:

- the robot moves from a starting position to the one of the requested part;

- after picking the part, the manipulator places it in a box, put at the starting point home;

- when all the parts corresponding to the first model are picked, the robot picks the parts corresponding to the following one;

- if the robot cannot reach any part on the vibratory plane, the feeder shakes it and, if there is not a sufficient number of distributed parts, it supplies more.

The placing position is defined as home, since it corresponds to the first position undertaken by the robot in order to not obstruct the sight of the camera. This position is set at the same height of viapoint, defined at $100 \mathrm{~mm}$ from the edge of the feeder: this was defined not only to avoid potential collision between the gripper and the feeder, but also to lower the cycle time by optimizing the robot trajectory. The other parameters that characterize the robot movement were defined in the Matlab script as shown in Table 1.

Table 1. Value for the motion parameter used in the test.

\begin{tabular}{cc}
\hline Parameter & Value (Unit) \\
\hline Speed & $100(\%)$ \\
Speed & $50(\%)$ \\
$H_{\text {appro }} /$ depart & $15(\mathrm{~mm})$ \\
offset from feeder & $1.5(\mathrm{~mm})$ \\
Viapoint $(x, y, z)$ & $420,214,140 \mathrm{~mm}$ \\
Viapoint $(\alpha, \beta, \gamma)$ & $0^{\circ} 180^{\circ} 0^{\circ}$ \\
home $(x, y, z)$ & $460,-102,140 \mathrm{~mm}$ \\
home $(\alpha, \beta, \gamma)$ & $0^{\circ} 180^{\circ} 0^{\circ}$ \\
\hline
\end{tabular}

It should be noted that these parameters could be set in the controller of the robot in an easier way, however in order to increase the flexibility of the system without the code becoming more complex, it was preferable to send them via the TCP-IP connection.

Since the joint 6 was sweeping a wide angle in a short linear movement, thus increasing the cycle time, its rotation at the viapoint was set equal to $60 \%$ of the rotation at the picking point. Moreover, since the parts could be reached with two different rotations due to the symmetry of the gripper, the value of the joint 6 at the picking point has been chosen as the nearest to zero; these solutions resulted in a decrease of the cycle time of about $10.6 \%$. The experimental picking time has been 
evaluated by means of a 103 test, as shown in Figure 4; each time interval was measured by Matlab since the instant the coordinates are sent to the controller to the one when the controller has notified Matlab that the robot has returned to the home position. The medium value results equal to $1.41 \mathrm{~s}$; an analysis of the distribution of the measured data, Figure 5, showed an interval of estimation equal to $\pm 0.12 \mathrm{~s}(95 \%)$.

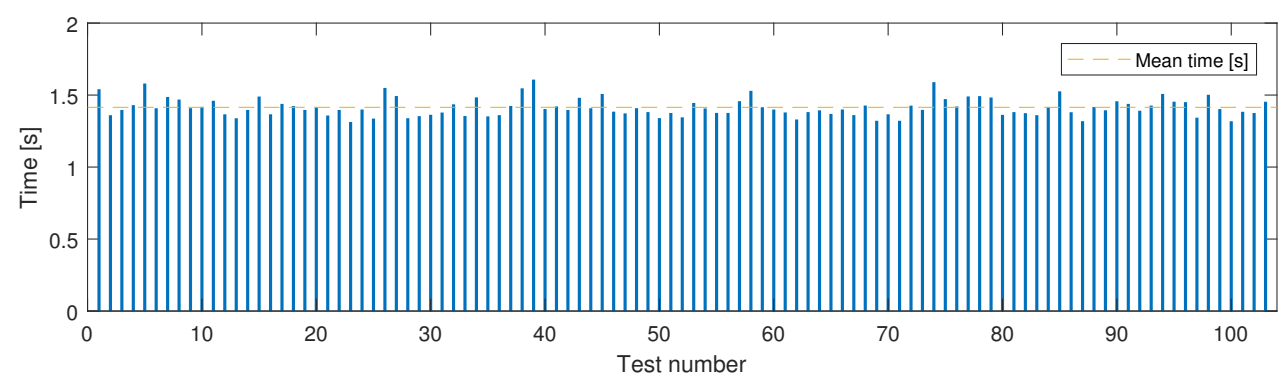

Figure 4. Measured time for each picking during the experimental test.

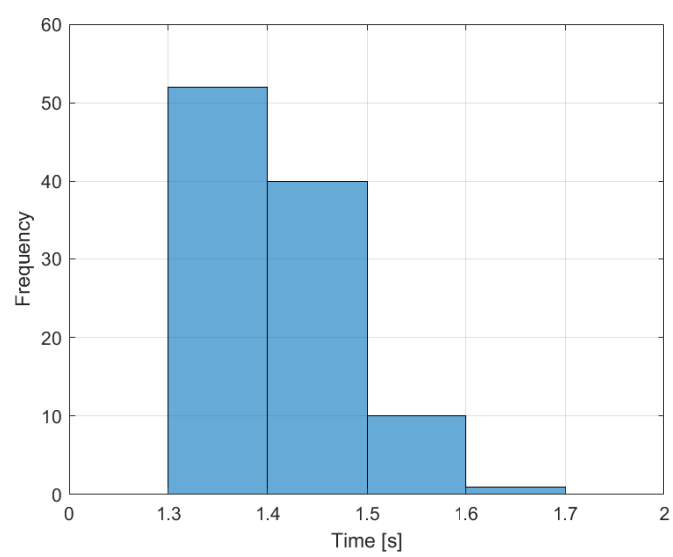

Figure 5. Frequencies histogram for the measured time.

\subsection{Hoppers Sequence Model}

The system considered is generally composed of an input belt, a suitable sequence of hoppers and an output belt. In this paper, we just focus on these devices, assuming that the system is fed by a bowl feeder with limited capability that delivers the kits to a packing machine. The maximum throughput of the feeder is set to 199.8 pieces/min, measured from the case study line. The motion of the pieces between a device and the subsequent is assumed to be a free falling motion, and is assumed that the discharging feed rate is infinite, that is, the pieces move together as a bulk from one hopper to its successor. The falling time can be so calculated as:

$$
T_{c}=\sqrt{\frac{2 \cdot h}{g}}
$$

where $h$ is the distance between two devices, here set to $200 \mathrm{~mm}$ and $g$ the gravity acceleration, assumed $9.81 \mathrm{~m} / \mathrm{s}^{2}$. We assumed that the pieces are delivered evenly spaced of a distance $d$ by the superior belt with speed $v_{\text {belt }}$, so that the time delay between two subsequent pieces falls is:

$$
T_{d}=\frac{d}{v_{b e l t}}
$$

The time between the opening command and the following idle state of a hopper is calculated assuming that the hopper can close after a percentage $p$ of the space between the hoppers has been 
covered by the falling components. The percentage here is set equal to $20 \%$, for a value of $d=200 \mathrm{~mm}$, means a distance of $40 \mathrm{~mm}$. Substituting this value into Equation (1), the time between the open command and the successive idle state is evaluated as:

$$
T=\cdot T_{a}+\sqrt{p} \cdot T_{\mathcal{C}}
$$

In order to find the filling time of the first hopper we use the values calculated with Equations (1) and (2):

$$
T_{f}=n \cdot T_{d}+T_{c}
$$

where $n$ is the number of components per kit delivered by the subline.

The values which could not be evaluated were obtained from the state of the art. Table 2 shows the values for the parameters used in the tests, where $T_{w}$ is the weighing time and $T_{m o v}$ is the time required to the inferior belt to shift of one position to another.

Table 2. Variable values taken into account for the simulation.

\begin{tabular}{cc}
\hline Parameter & Value (s) \\
\hline$T_{a}$ & $0.15-0.25-0.32$ \\
$T_{c}$ & 0.202 \\
$T_{\text {mov }}$ & $0.3-0.6$ \\
$T_{w}$ & 0.8 \\
Configuration & $3-2-2 \times 2$ \\
\hline
\end{tabular}

Three configurations reported in Figure 6, were considered to perform the study of the hoppers opening sequence:

- The first configuration is composed of three hoppers. The first is the pool hopper, the second is a weighing hopper to perform the quality inspection and the third is a boost/memory hopper. This layout will be referred as the 3 configuration. It is depicted in Figure 6 .

- The second configuration is composed of two hoppers. A pool hopper and a boost/memory one. The quality inspection is performed by a camera set upon the superior belt. This layout will be referred as the 2 configuration. It is depicted in Figure $6 \mathrm{~b}$.

- The third configuration is composed of two columns with two hoppers each and a selector between the superior belt and the hoppers. The quality inspection is performed by a camera set upon the superior belt. This layout will be referred as the $2 \times 2$ configuration and is depicted in Figure 6c.

The proposed case study inspired the 3 configuration, which is assumed to be the starting point of the study. In this configuration, the weighing hopper is needed to perform the quality inspection, verifying if the correct number of pieces has been delivered by the superior belt, in order to avoid defective kits in output. Such weighing device is equipped with a load cell used to estimate the weight, which provides a raw electrical signal. The main constraint in achieving high accuracy is a superimposed noise, which is eliminated thanks to a filter. However a trade-off between the measurement time and a high precision estimation has to be accepted. Thus the weighing time needed to obtain a stable measurement tends to become the bottleneck of the system, preventing it from reaching high efficiency and saturating the input devices. The 2 configuration addresses the problem eliminating the weighing device and replacing it with a vision system, capable of performing in its place the quality inspection. This solution is depicted in Figure $6 \mathrm{~b}$, and since it does not contemplate the use of a weighing device, it is not affected by the aforementioned trade-off, allowing to perform a $100 \%$ quality control ignoring the weighing time and to discard single components instead of a full batch recognized as defective by the weighing device at the end of the sequence. 


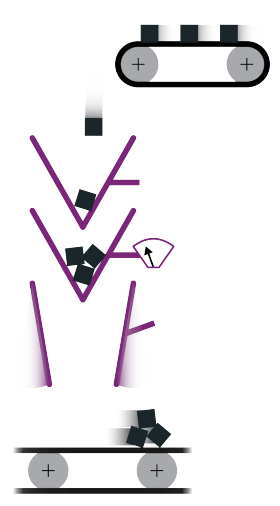

(a) Layout 3

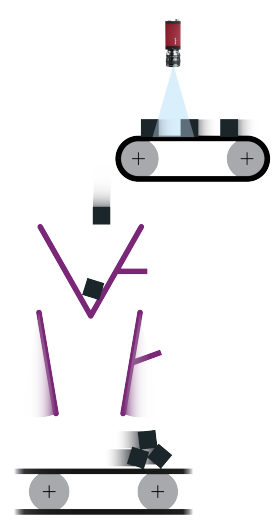

(b) Layout 2

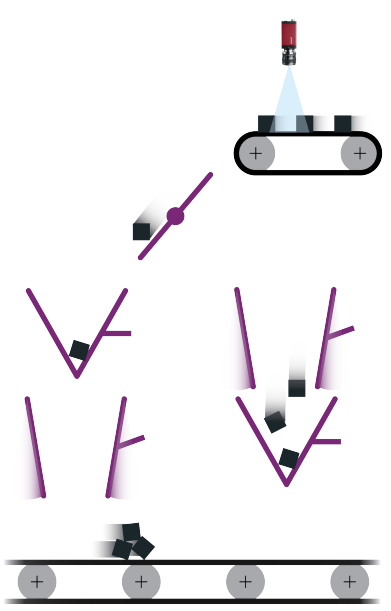

(c) Layout $2 \times 2$

Figure 6. Layouts considered.

The throughput increase in the simulation was relevant, but the input feeder could not be saturated. Therefore we developed the $2 x 2$ configuration depicted in Figure $6 c$, which is capable of saturating the input feeder, thanks to a higher throughput.

In order to find the throughput of the system, the bottleneck of each configuration was analyzed and its takt time described with equations. In the 2 configuration the bottleneck is the first hopper, whose takt time is:

$$
\text { TaktTime }=T_{a}+(1+\sqrt{p}) \cdot T_{c}+n \cdot T_{d}
$$

In the 3 configuration the bottleneck is the weighing hopper and a superior limit is set by the takt time of the 2 configuration:

$$
\text { TaktTime }=\max \left\{\begin{array}{c}
T_{a}+(1+\sqrt{p}) \cdot T_{c}+n \cdot T_{d} \\
T_{a}+T_{w}+(1+\sqrt{p}) \cdot T_{c}
\end{array}\right.
$$

In the $2 x 2$ configuration the bottleneck is the first hopper, which is filled by the belt:

$$
\text { TaktTime }=n \cdot T_{d}+T_{c}
$$

A superior limit for all the configurations is set by the inferior belt whose minimum takt time is:

$$
\text { TaktTime }=T_{c}+T_{m o v}
$$

From these equation is possible to calculate the throughput of the system for each configurations with different parameters with the following equation:

$$
Q=\frac{1}{\text { TaktTime }}
$$

Figure 7a shows the throughput for different number of parts and for each studied configuration using parameters in Figure 7. As expected, increasing the number of parts for each kit decreases the throughput, since $n \cdot T_{d}$ increases. It should be noted that the $2 \times 2$ configuration results in the maximum throughput compared to the others. 


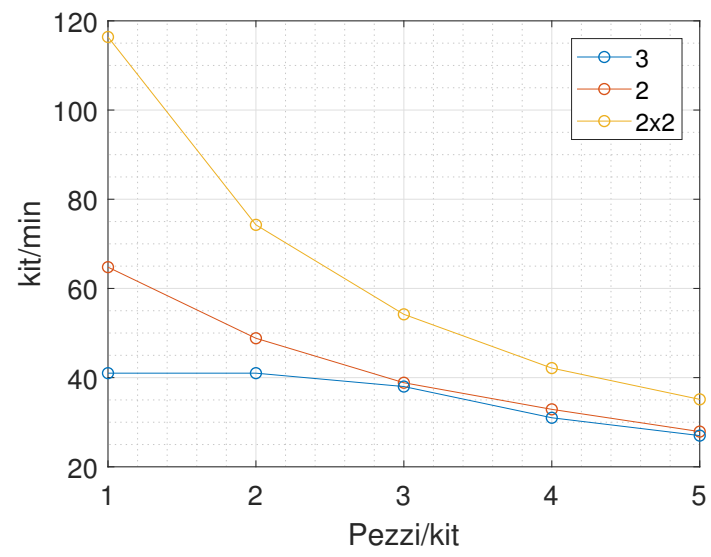

(a) Plot of the modelled throughput of the configurations

\begin{tabular}{c|c}
\hline Parameter & Value [s] \\
\hline$T_{a}$ & 0.32 \\
$T_{c}$ & 0.2 \\
$T_{d}$ & 0.3 \\
$T_{w}$ & 0.8 \\
$T_{\text {mov }}$ & 0.3 \\
\hline
\end{tabular}

(b) Parameters used for the modeled throughput in the presented figure

Figure 7. Modeled throughput of the configurations.

\subsubsection{Sensitivity Analysis}

A sensitivity analysis was carried out in order to observe the sensitivity of the presented model to different values for the considered parameters. Since $T_{a}, T_{c}, T_{d}$ and $T_{w}$ were obtained from the state of the art, we believe that they represent a source of uncertainty; therefore, we tested the system behavior for different values of the aforementioned parameters. Following Equations (5)-(7), a test was run by changing the values as represented in Table 3, following an One-at-a-time approach.

Table 3. Values considered for the parameter of the sensitivity analysis.

\begin{tabular}{cc}
\hline Parameter & Value (s) \\
\hline$T_{a}$ & $0.10-0.30-0.40$ \\
$T_{c}$ & $0.14-0.20-0.24$ \\
$T_{d}$ & $0.20-0.30-0.40$ \\
$T_{w}$ & $0.50-0.80-1.00$ \\
\hline
\end{tabular}

The plots in Figure 8 are obtained by evaluating the takt time with the aforementioned model for the different values.

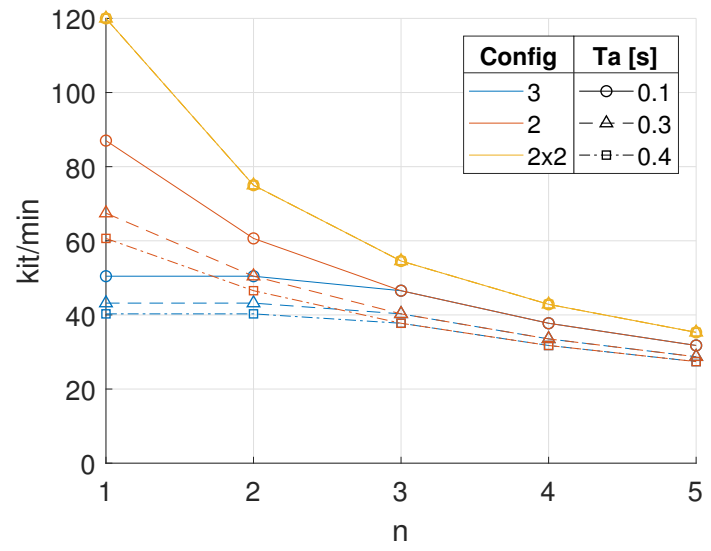

(a) Sensitivity analysis for $T_{a}$

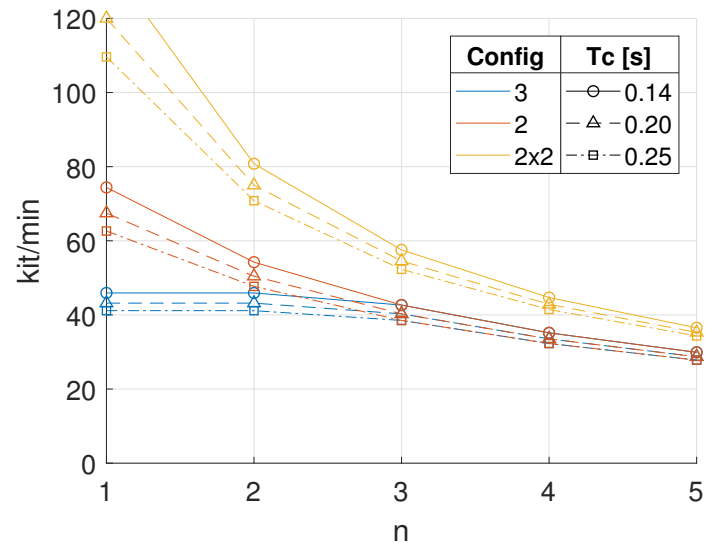

(b) Sensitivity analysis for $T_{\mathcal{C}}$

Figure 8. Cont. 


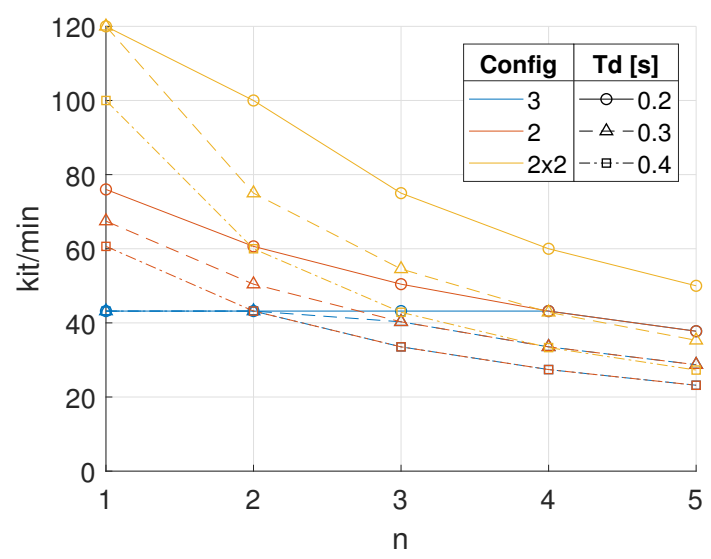

(c) Sensitivity analysis for $T_{d}$

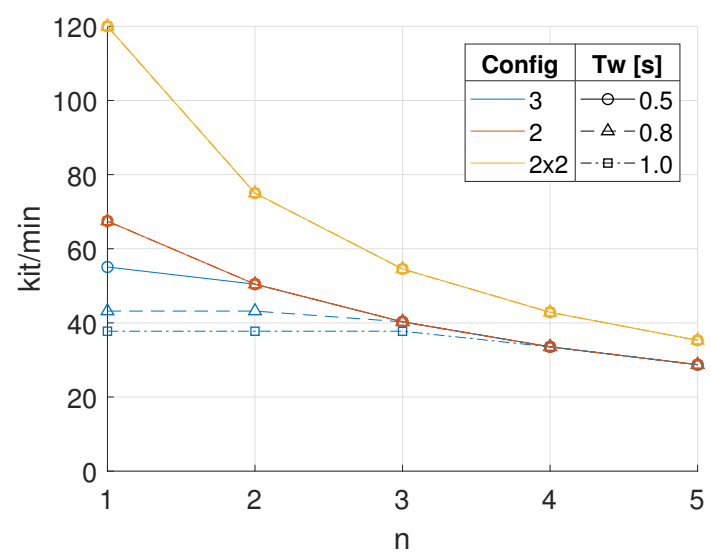

(d) Sensitivity analysis for $T_{w}$

Figure 8. Results of the sensitivity analysis for the parameters $T_{a}, T_{c}, T_{d}, T_{w}$.

\subsubsection{Simulation}

A simulation of the configurations was performed with a finite state machine, which uploads the states of the different devices over time. Since the simulation is affected by transient and/or random effects, considered as random variations on the parameters, it could be used as a reference for the proposed model; moreover, it provides for further data, such as the task scheduling and the availability of the feeders. Each device is linked to its predecessor and its successor with the relationships illustrated in Figure 9, which describes the transition between states. The superior belt is the input device of the system and switches just between two states, ready and filling, with a logic described in Figure 9a. It interacts only with the first hopper, so its state depends only on the state of this device. The generic hopper switches between many states, filling, idle, weighing, ready, closing and opening. The non weighing hopper presents the same graph without the state weighing. The generic hopper can be related to one other hopper or a belt, referred as predecessor if it is in a previous position on the feeding chain or successor if it is in a next position on the feeding chain. Its graph is depicted in Figure 9c. The inferior belt, that is, the output device of the system, can switch between the states filling, moving and idle. The moving state is due to the need of simulate the time required from the output belt of the case study line to switch between two subsequent position. The states are described in detail in Table 4. Each transition is due to the change of state of an adjacent device or for the expiration of the time needed for a specific action (e.g., the opening and closing movement of a hopper).

The transition logic just described was implemented into a Matlab script in order to perform time varying simulations of the system. To reflect the effect of the uncertainties on the process in a real environment we introduced some random variation of the parameters of the simulation. A random variation in the interval $[-0.1 \mathrm{~s},+0.1 \mathrm{~s}]$ was added to $T_{w}$ to simulate the dynamic effect of the components falling on the weighing hopper and a Gaussian distribution ( $\mu=100 \mathrm{~mm} ; \sigma=15 \mathrm{~mm}$ ) of the components spacing on the input belt was assumed to simulate some variability in the bowl feeder feeding rate.

Table 4. The states implemented for the devices in the simulation.

\begin{tabular}{ll}
\hline States & Description \\
\hline Ready & The device is ready, but its successor not \\
Idle & The device is ready, but its predecessor not \\
Opening & The hopper is opening \\
Closing & The hopper is closing \\
Filling & The hopper or the inferior belt is being filled, or the superior belt is filling \\
Weighing & The weighing hopper is performing the weight measure \\
Moving & The inferior belt is shifting to the next position \\
\hline
\end{tabular}




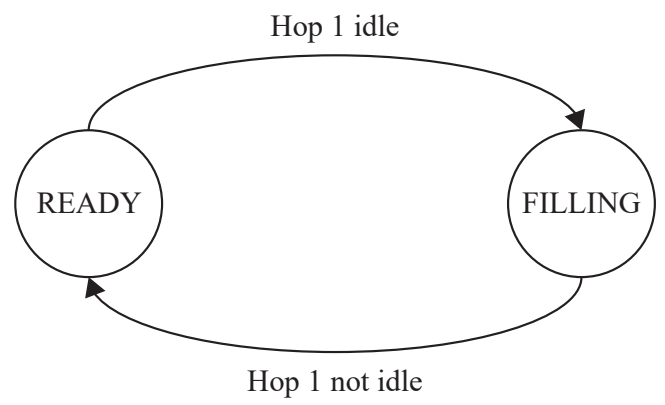

(a) Superior belt graph

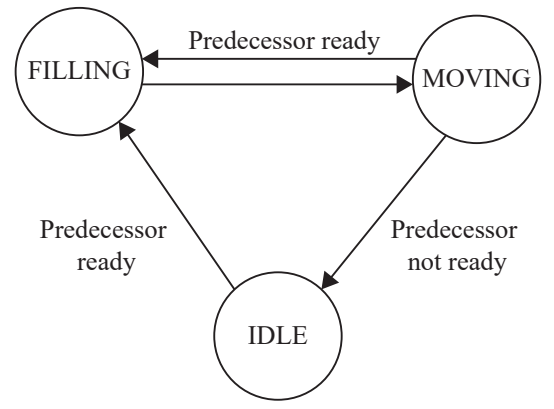

(b) Inferior belt graph

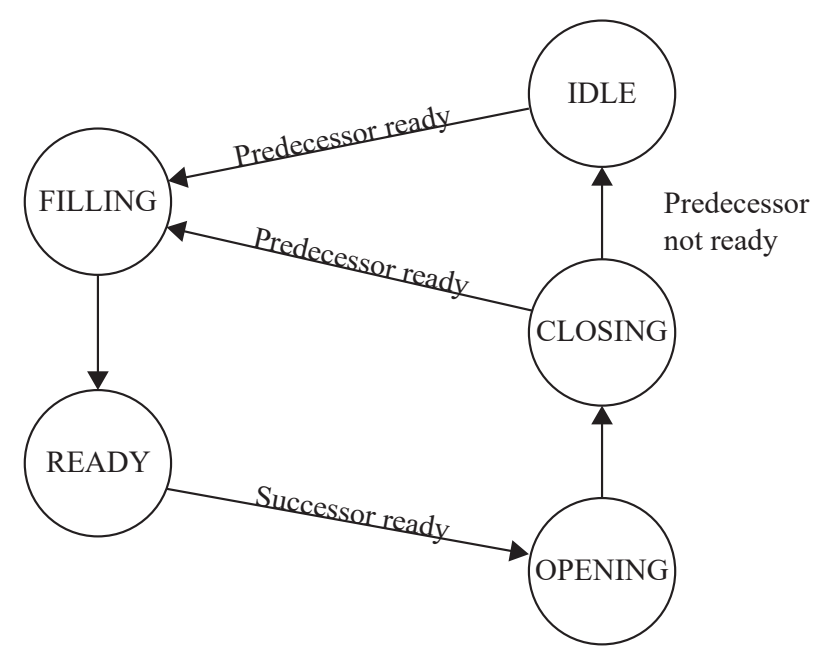

(c) Hopper graph

Figure 9. Finite state graphs of the devices.

A further simulation with the same parameters in Figure 7 was performed and the throughput for the three configurations is shown in Figure 10. The relative error between the model prediction and the simulated throughput was calculated and presented in Table 5. The values state a good agreement between the model and the simulation, except for the tendency of overestimate the throughput, which can be seen from the negative mean error.

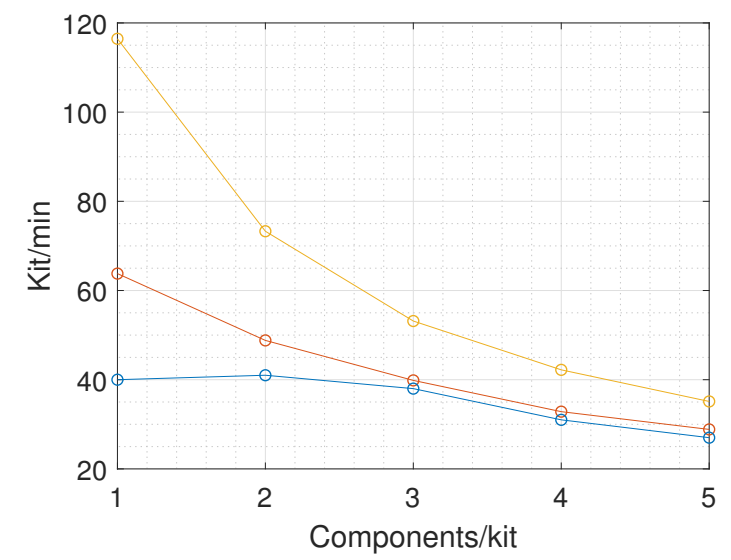

Figure 10. Plot of the throughput found with the simulations. 
Table 5. Relative error (\%) between the model prediction and the simulated throughput.

\begin{tabular}{ccccccc}
\hline \multirow{2}{*}{ Configuration } & \multicolumn{7}{c}{$\mathbf{n}$} & \\
\cline { 2 - 6 } & $\mathbf{1}$ & $\mathbf{2}$ & $\mathbf{3}$ & $\mathbf{4}$ & $\mathbf{5}$ & \\
\hline 3 & -6.04 & -3.69 & -4.40 & -6.51 & -5.08 & -5.14 \\
\hline 2 & -3.31 & -1.61 & 0.24 & -0.98 & 1.44 & -0.84 \\
\hline $2 \times 2$ & -2.96 & -2.28 & -2.55 & -1.51 & -0.48 & -1.96 \\
\hline
\end{tabular}

One output of the simulation is the state of any device in every moment, which could be used to plot the state chart of the devices, like the one reported in Figure 11, plotted for the values $T_{a}=0.15 \mathrm{~s}$, $T_{\text {mov }}=0.30 \mathrm{~s}$ and $n=5$.

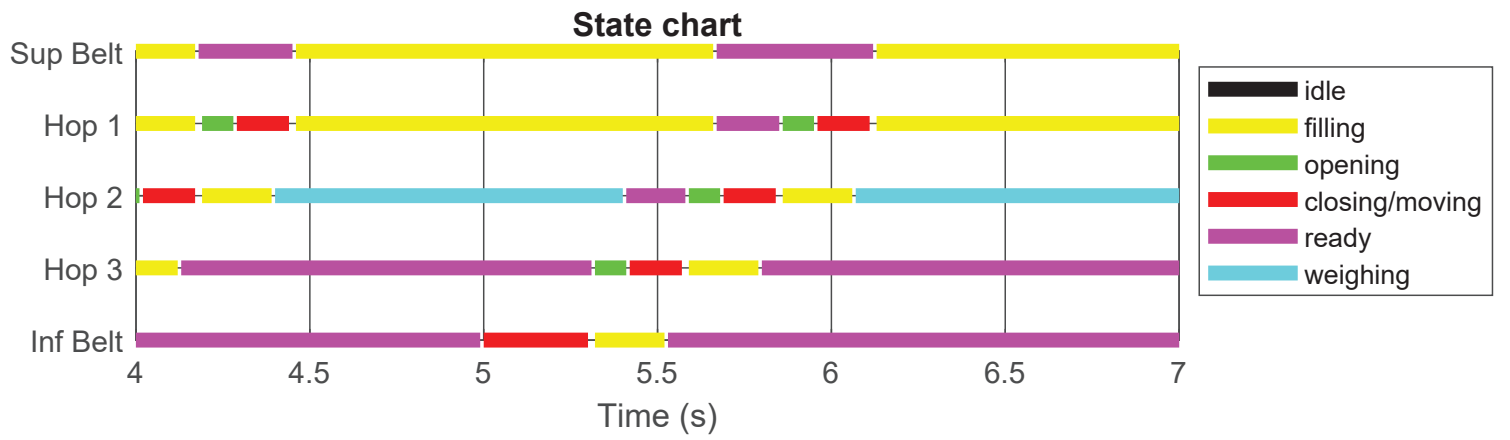

Figure 11. An example of state chart of the devices obtained with the simulation.

The availability is the ratio of run time to planned production time. With the data used to plot such state charts was also possible to evaluate the availability for the devices as:

$$
A=\frac{R T}{P P T}
$$

where $A$ is the availability, $R T$ is the run time, i.e., the time the device did not spend in ready or idle state, and PPT is the planned production time, i.e., the total time the device is used.

In Figure 12 we can see the results of the simulations performed varying the opening and closing time of the hoppers to understand their influence on the system. To the left the data calculated with a $T_{a}=0.15 \mathrm{~s}$, while to the right the data related to $T_{a}=0.32 \mathrm{~s}$. The resulting plot shows that the opening time of the hoppers slightly affects the performance of the system, which decreases with low kit size, due to the increasing frequency of opening/closing operations. The worsening interests mainly the 3 and 2 configurations, but not change substantially the comparison between the configurations, therefore from now on we will refer indistinctly to the plot to the left or to the right column in Figure 12. The plot Figure 12b shows that the $A$ of the superior belt for the $2 \times 2$ configuration (yellow solid line) is 1 for any kit size considered. It should be noted that only the $2 x 2$ configuration is able to reach this value, which mean the saturation of the superior belt. For a kit size equal to 1 the inferior belt is close to saturation. From the plot in Figure 12d we can see that the kit throughput of the $2 x 2$ configuration rises with the decrease of the kit size. Figure $12 \mathrm{f}$ is the product of the kit throughput, that is, the plot in Figure $12 \mathrm{~d}$, and the kit size.

The $A$ of the superior belt for the 2 configuration, as can be seen in plot Figure 12b, starts from values near to 0.82 and drops for decreasing kit size. From the plot Figure $12 \mathrm{~d}$ is clear that its performance is worse than the $2 x 2$ configuration but outperforms the 3 configuration for a low kit size ( 1 and 2). Finally the $A$ of the superior belt for the 3 configuration, as can be seen from the plot Figure $12 \mathrm{~b}$, is similar to the one of the 2 configuration, but for decreasing kit size it shows a heavier drop. From the plot Figure 12d is clear that for a kit size lower or equal than 2 the throughput of the system is stable, due to the saturation of the weighing feeder. This means a reduction of component 
throughput, as can be seen from plot Figure 12f. It is to be noticed that all the plots in Figure 12 are of minor importance for small kit sizes, due to the assumption made in the introduction that the feeding of low quantity components is addressed with the development of a flexible robotic workcell.

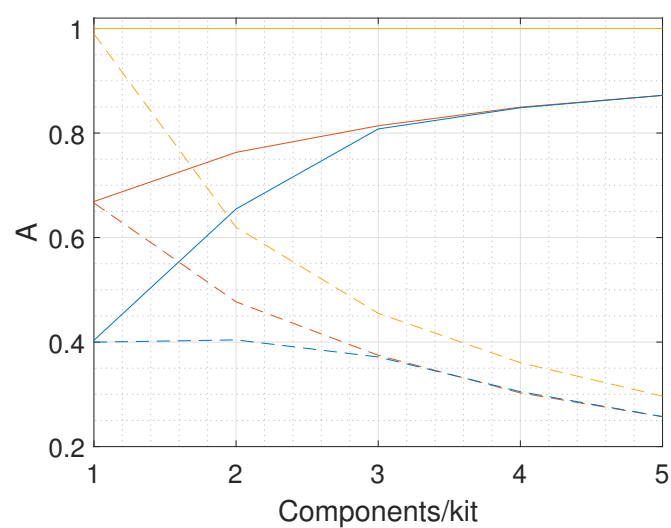

(a) A, Ta $=0.15 \mathrm{~s}$

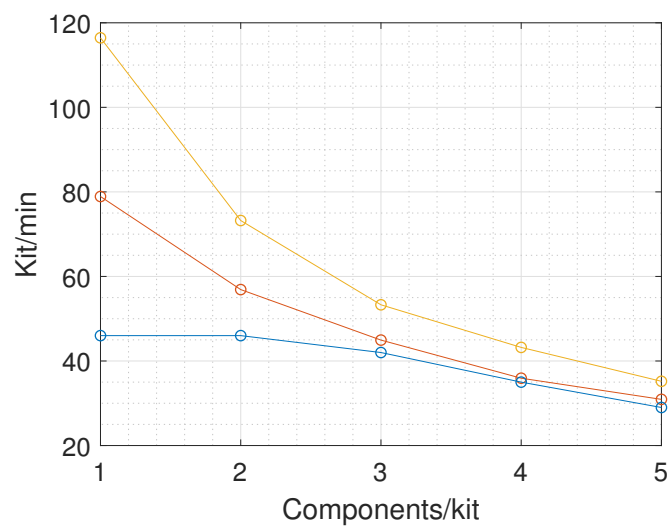

(c) $\mathrm{kit} / \mathrm{min}, \mathrm{Ta}=0.15 \mathrm{~s}$

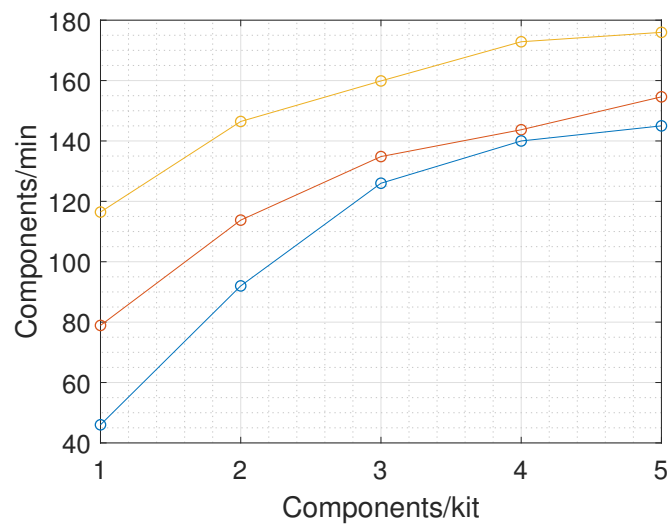

(e) pieces $/$ min, $\mathrm{Ta}=0.15 \mathrm{~s}$

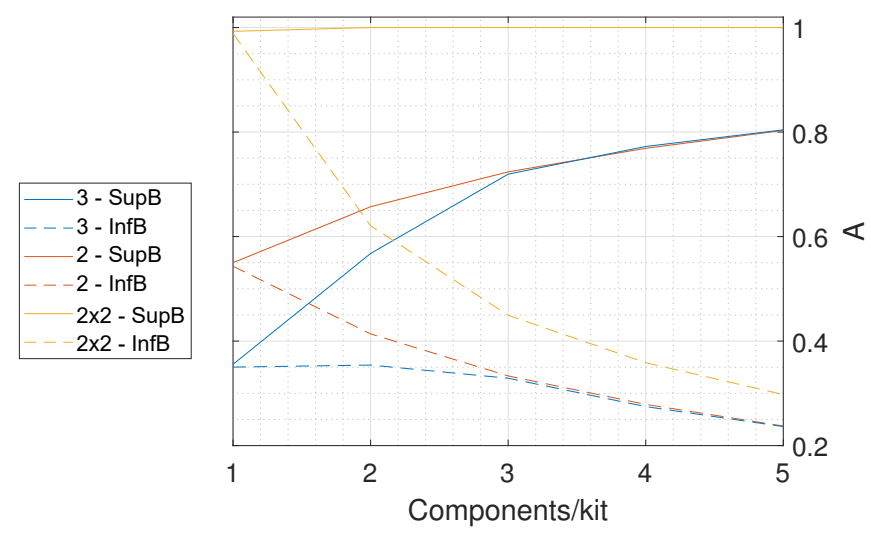

(b) $\mathrm{A}, \mathrm{Ta}=0.32 \mathrm{~s}$

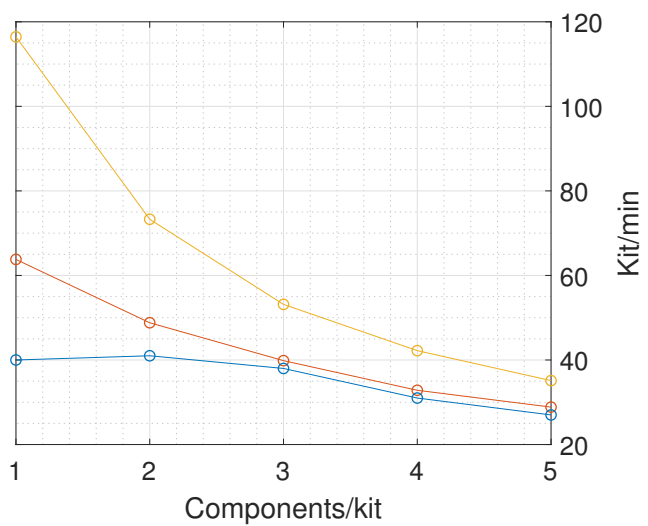

(d) kit $/ \mathrm{min}, \mathrm{Ta}=0.32 \mathrm{~s}$

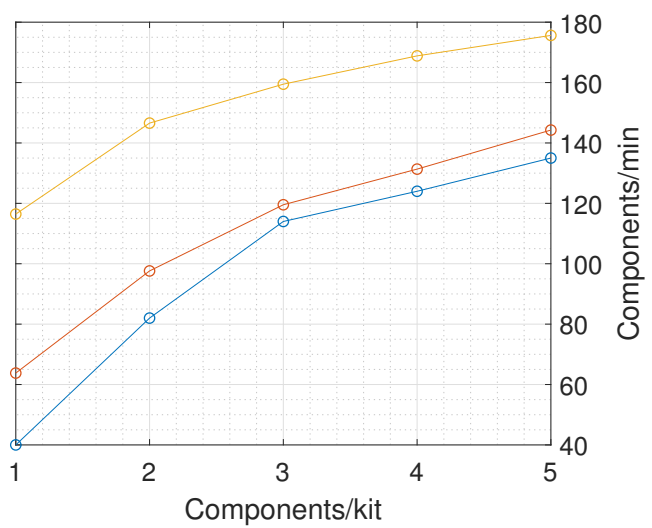

(f) pieces $/ \min , \mathrm{Ta}=0.32 \mathrm{~s}$

Figure 12. Throughput and availability of the system varying the opening and closing time of the hoppers.

Figure 13 depicts the behavior of the system for different $T_{\text {mov }}$. The left column of plots is defined for $T_{m o v}=0.30 \mathrm{~s}$, while the right column is defined for $T_{m o v}=0.60 \mathrm{~s}$, the last taken from the case study line. From Figure $13 \mathrm{~b}$ it is clear that the $2 \times 2$ configuration is strongly affected by a slower belt and its $A$ drops for a kit size smaller than one. The dashed yellow line shows that for that kit size the inferior belt starts to be saturated and cannot accept higher throughput, causing a decrease in the $A$ of 
the previous devices. It is noticeable that the 3 and the 2 configuration in the two cases are almost not affected by the time required by the inferior belt to perform a shift, as we can see from the comparison between Figure 13c,d.

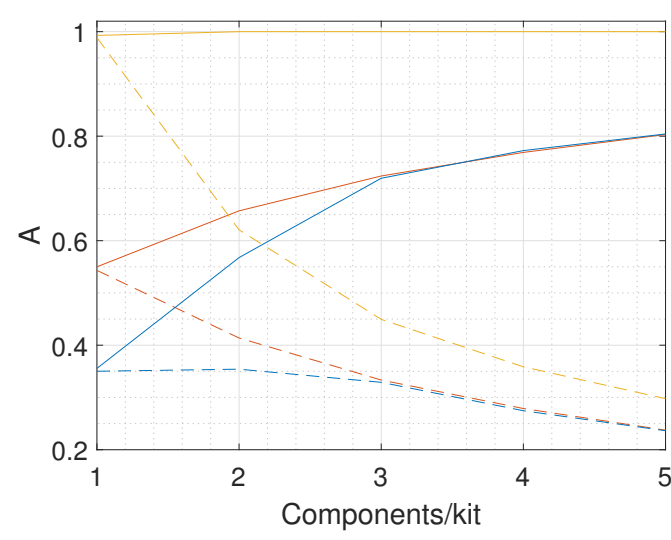

(a) $\mathrm{A}$, Tmov $=0.30 \mathrm{~s}$

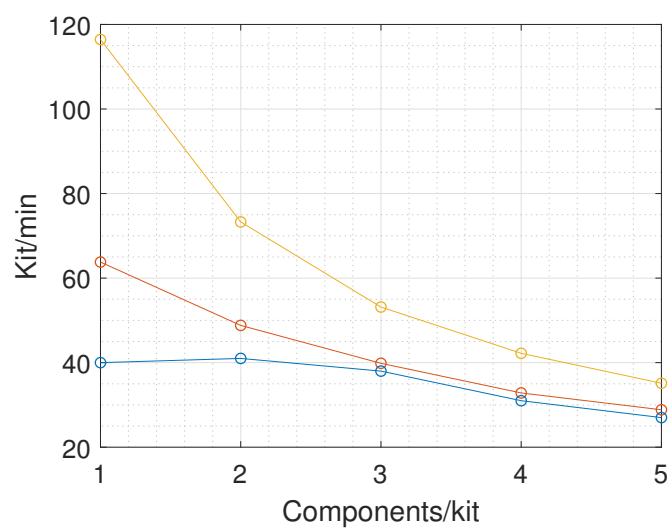

(c) $\mathrm{kit} / \mathrm{min}, \mathrm{Tmov}=0.30 \mathrm{~s}$

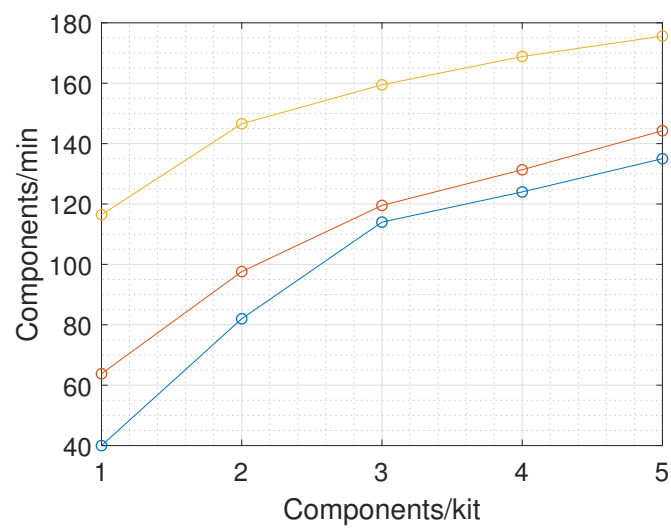

(e) pieces $/$ min, Tmov $=0.30 \mathrm{~s}$

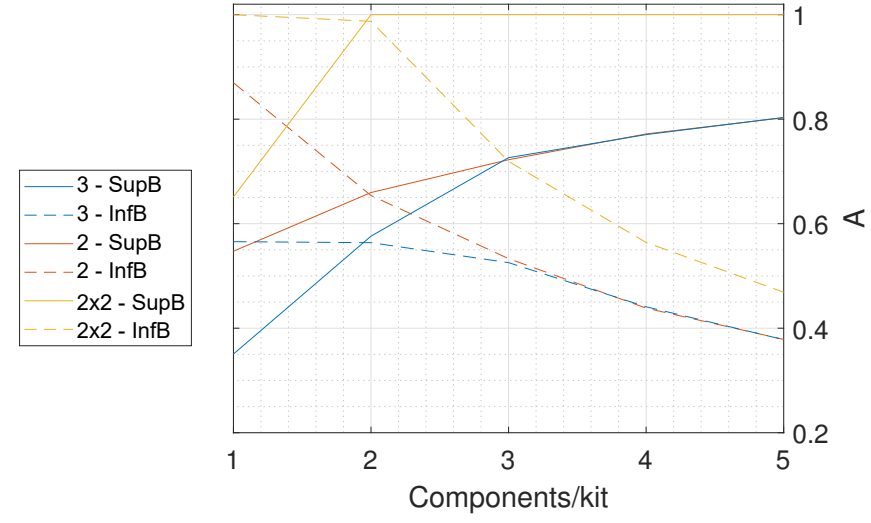

(b) A, Tmov $=0.60 \mathrm{~s}$

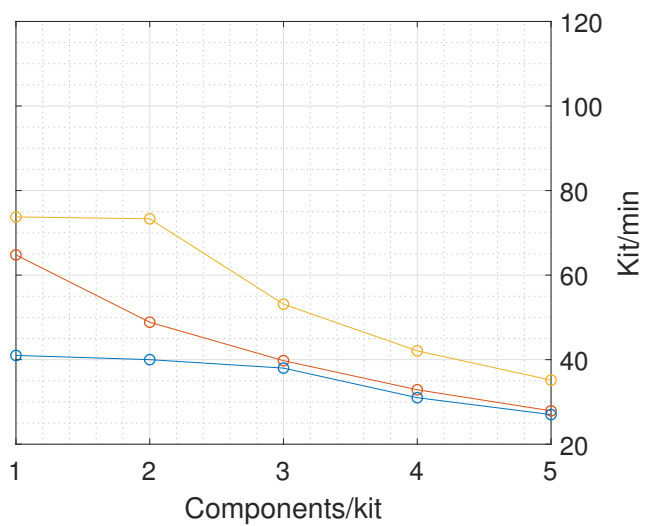

(d) kit $/ \mathrm{min}, \mathrm{Tmov}=0.60 \mathrm{~s}$

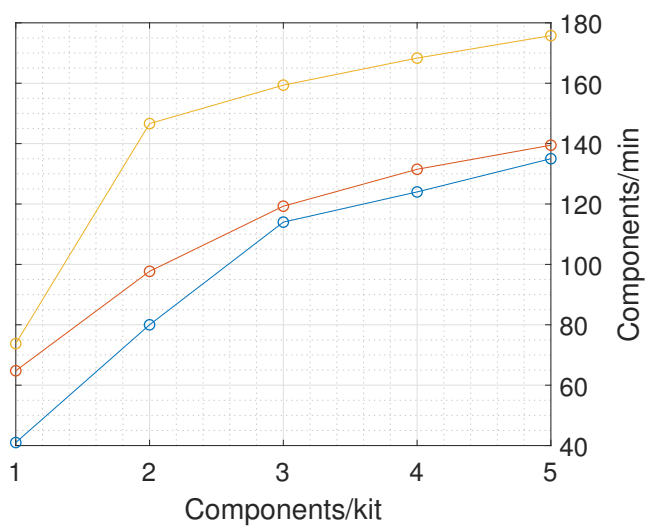

(f) pieces $/ \mathrm{min}, \mathrm{Tmov}=0.60 \mathrm{~s}$

Figure 13. Throughput and availability of the system varying the time required to the inferior belt to shift to one position to another.

It is important to notice that the $2 x 2$ configuration would be capable of feed two inferior belts as depicted in Figure 1, so that the $T_{\text {mov }}$ would be approximately half compared to the other two configurations. The comparison between the configurations would make the $2 \times 2$ configurations even more valuable. 


\subsubsection{Vision Inspection System}

In the Industrial Robotics Laboratories at the University of Padua a prototype of the vision quality inspection system placed onto the superior belt was developed. The system is composed by a AVT Pike F032- $b$ camera, triggered by a Sick DRS61 incremental encoder. The belt's width is $400 \mathrm{~mm}$, its speed is set equal to $200 \mathrm{~mm} / \mathrm{s}$. The pieces are put on the conveyor belt and they run on the backlighted area, while the camera acquires images, as illustrated in Figure 14c.

The matrix Pike camera is used as a quasi-linear camera, acquiring 6 lines at a time. It is synchronized with the belt thanks to the encoder. The acquisition is performed by a $\mathrm{C}++$ algorithm which stores one hundred acquisition into a buffer and then send all the content of the buffer to Matlab, where is performed the image elaboration and then controlled the air blow to discard defective pieces.

The image processing consists in a sum of the pixel intensity value performed by row, obtaining the profile illustrated in Figure 14b. A check on the length of the piece was used to detect defects, but further works could lead to the development of more sophisticated methods, thanks to the clear profile of the objects obtained with this setup.

It is really important to notice that with the introduction of a vision inspection system that is able to discard the wrong or defective pieces, the retooling also becomes easier. In fact, bowl feeders require an amount of pieces higher than the actual production lot to be handled, in order to guarantee a uniform output flow [16]. This leads to high retooling time, due to the need to empty the feeder of the previous lots, before filling it with the new components. The vision inspection system could lead, together with the adoption of a flexible feeder, to a dynamic lot change, removing the need to empty the feeder and letting the vision inspection system discard the previous components.

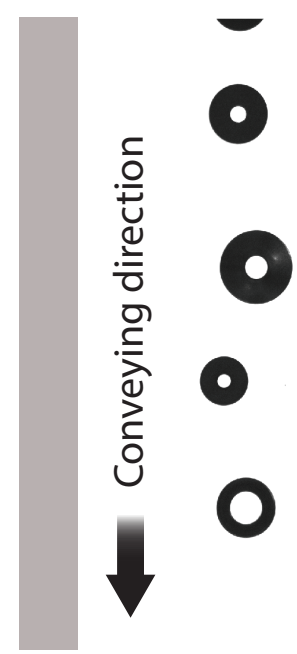

(a) Snaphshot of the camera.

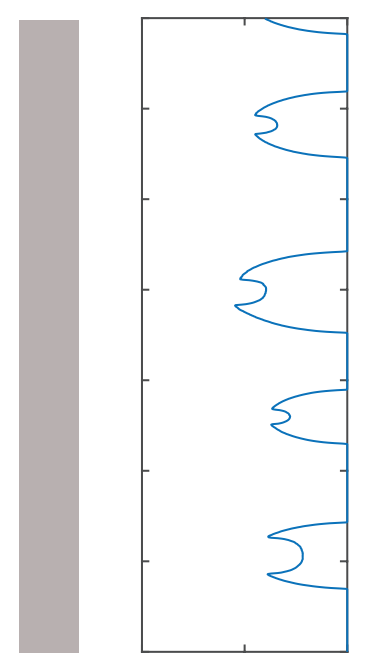

(b) Profile obtained from image processing.

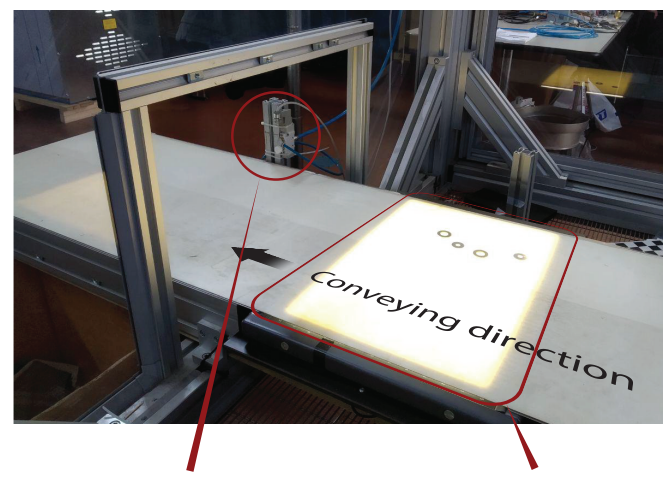

Air blow to discard Backlighted area for defective pieces camera acquisition

(c) Experimental setup.

Figure 14. Vision system experiment.

\section{Case Study}

To demonstrate the effectiveness of the proposed solutions, these were applied to the considered case study, with a specific focus on the hoppers cascade system model. The actual system, as described in Section 2.2 as system 3 and represented in Figure 15, is composed of three hoppers of which the middle one performs the weighing. The predictions of the proposed model were compared to the current solution, validating the expected results with the real data. Moreover the possible throughput improvements adopting the 2 and $2 \times 2$ configurations were evaluated. Table 6 shows the parameters used in the model. 
The feeders of the considered assembly kitting line feed the same number of components and $n$ will be equal to 2 or 4 . The comparison will then involve two values for each considered configurations, reported in Table 7.

The throughput obtained for the 3 configuration from the model, corresponding to the first row of Table 7, can be compared with that measured from the case study, which is equal to $22.05 \mathrm{kit} / \mathrm{min}$ and to $32.56 \mathrm{kit} / \mathrm{min}$, respectively for $n$ equal to 2 and 4 . The resulting error for the 3 configuration is therefore equal to $6.07 \%$ and $1.62 \%$. We believe therefore that it is possible to affirm that the model is validated by the case study; moreover it is clear how the $2 x 2$ solution is the most convenient one in terms of throughput, showing an increment in productivity of about $49.6 \%$, which potentially could lead to the removal of two bowl feeders.

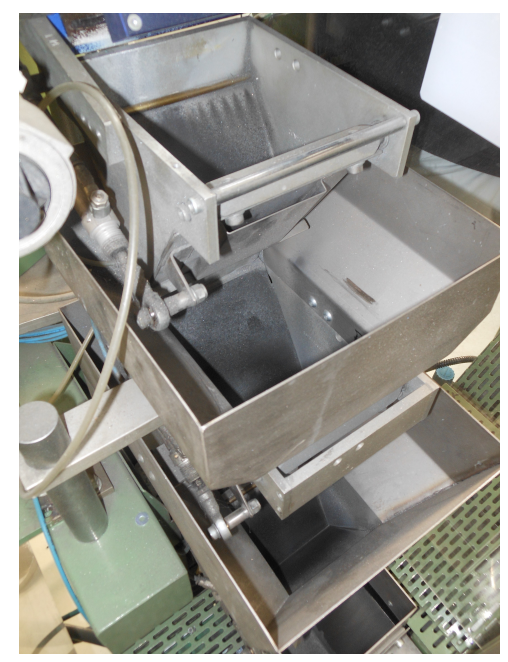

Figure 15. Case study hoppers cascade system. In this application the system is composed of three hoppers and a weighing device in the middle one.

Table 6. Values of the parameters for the case study.

\begin{tabular}{cc}
\hline Parameter & Value (s) \\
\hline$T_{a}$ & 0.36 \\
$T_{\mathcal{c}}$ & 0.28 \\
$T_{d}$ & 0.45 \\
$T_{w}$ & 1.0 \\
$T_{m o v}$ & 0.6 \\
$n$ & $2-4$ \\
\hline
\end{tabular}

Table 7. Throughput obtained from the model for the parameters taken from the case study.

\begin{tabular}{cll}
\hline \multirow{2}{*}{ Configuration } & \multicolumn{2}{c}{ Throughput (kit/min) } \\
\cline { 2 - 3 } & $\mathbf{n = 2}$ & $\mathbf{n = 4}$ \\
\hline 3 & 23.39 & 33.99 \\
2 & 23.39 & 36.03 \\
$2 x 2$ & 28.85 & 50.85 \\
\hline
\end{tabular}

\section{Results Discussion}

Since the model has been verified, the impact on the efficiency of the assembly kitting line can be evaluated. The introduction of the flexible feeder device allows to remove two bowl feeders in the case study considered, the ones designated to supply the parts with low quantity for kit. Moreover, the model of the hoppers of the rigid feeders, as stated by the analysis of the case study, allowed to remove two more, therefore the resulting H-FAS is composed by: 
- four rigid feeders with hoppers devised to supply the high volume components as described before;

- one fully flexible feeder to supply low volume components;

- one manipulator in charge of picking the part from the flexible feeder.

In order to evaluate the improvements of the proposed solution, the OEE was chosen as a proper parameter. This is defined as the product of three different values: the availability, that takes into account any events that stop the planned production, the quality, i.e., the number of parts that does not match the requirements, and the performance, i.e., the devices speed.

The first parameter considered, the availability $D$, was evaluated as:

$$
D=\frac{T_{\text {batch }}}{n \cdot T_{\text {setup }, i} \cdot T_{\text {batch }}}-0.05
$$

where $T_{\text {batch }}(\mathrm{h})$ is the production time for the single batch, $T_{\text {setup }, i}(\mathrm{~min})$ is the time needed for the setup of each of the $n$ feeders, such as $T_{\text {setup }, i}$ times $n$ equals to the total $T_{\text {setup }}$. We considered $n$ equal to 8 in the original case and 4 in the proposed development, since the flexible feeder does not need any setup. Lastly, the 0.05 value is a corrective constant defined in order to consider potential unexpected stops. Figure 16 represents the percentage difference of $D$ between the original system and the proposed one in case of different values of $T_{\text {batch }}$ and $T_{\text {setup }}$.

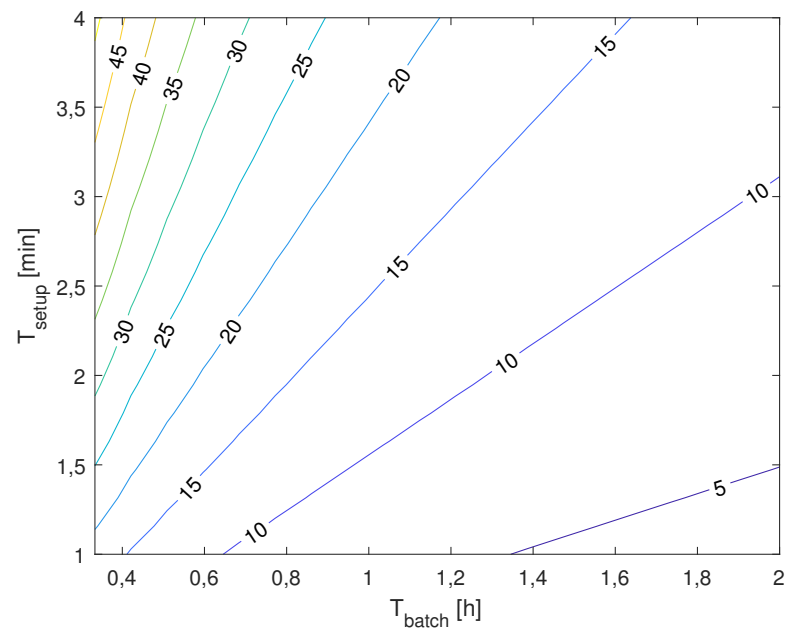

Figure 16. Availability increase (\%) by changing the number of re-configurable feeders.

For the quality and performance parameter, a different equation was needed. Defined $P_{d}$ the probability of defects and $P_{b}$ the probability of slow cycles for each feeder considered, we could define the total quality parameter $Q$ and the total performance parameter $P$ as

$$
\begin{aligned}
& Q=1-P_{D} \\
& P=1-P_{B}
\end{aligned}
$$

where $P_{D}$ and $P_{B}$ are evaluated as stated by the law of total probability [17] applied for $n$ equal to 8 and 4 feeders:

$$
\begin{aligned}
& P_{D}=P_{d} \cdot n+P_{d}^{n}-\left(\sum_{k=2}^{n-1}(-1)^{k}\left(\begin{array}{l}
n \\
k
\end{array}\right) \cdot P_{d}^{k}\right) \\
& P_{B}=P_{b} \cdot n+P_{b}^{n}-\left(\sum_{k=2}^{n-1}(-1)^{k}\left(\begin{array}{l}
n \\
k
\end{array}\right) \cdot P_{b}^{k}\right)
\end{aligned}
$$


The range value used for $P_{d}$ is restrained between $0.05 \%$ and $0.5 \%$, while the values of $P_{b}$ are evaluated as 0.7 times $P_{d}$, based on the data from the case study. Figure 17 represents the difference of the product of $Q$ and $P$ evaluated for $n$ equal to 8 and 4 in the case of different values of $P_{d}$ and $P_{b}$ defined from the examined case study in the considered range.

It is possible to notice a significant increment in the OEE and therefore in the throughput, especially in the availability parameter, which could reach an increase of over $40 \%$. This proves the starting hypothesis that the proposed reconfiguration of the feeding system in the case study kitting assembly process could improve the throughput.

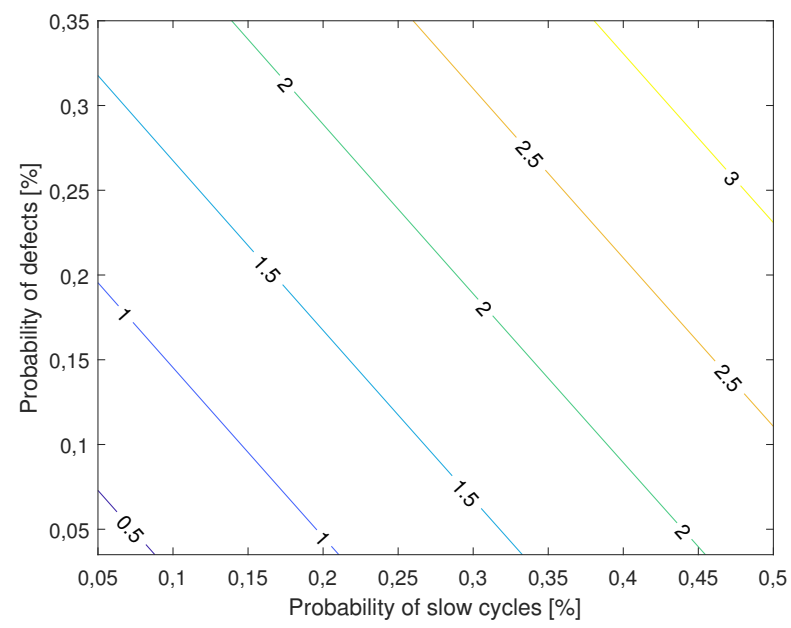

Figure 17. Quality and performance increase (\%) by changing the number of re-configurable feeders.

\section{Conclusions}

We presented a solution in order to increase the efficiency of an assembly kitting line based on an H-FAS system. Firstly, we noticed that a low quantity of components could be supplied by a fully flexible feeder, thus reducing further the number of vibratory feeders. An F-FAS subsystem was studied in the laboratory in order to test the performance of the system in a kitting assembly line.

With regards to the high quantity components, the current bowl feeders could be preserved, re-configuring only the hoppers cascade system. Several studies have been proposed in this field of research; however, they are focused on the material characteristics and not on the the system itself; thus, our work suggested an innovative solution based on several reconfigurations of the feeding system. A mathematical model has been developed and its robustness was verified by means of a sensitivity analysis; moreover, we developed a simulation to evaluate the accuracy of the proposed model, since the simulation is a finite state machine which performs by uploading the states of the different devices.

We noticed that, for a certain range of opening time, the throughput is not so much influenced; we believe that rising the speed of the lower belt could present further improvements for the alternative configurations developed from 3 . The requested weighing device can be replaced with the adoption of a vision system, similar to the one suggested in this work. This was tested in the laboratory and showed an initial but effective solution for quality inspection.

Lastly, we evaluated the increased throughput of the system by calculating the percentage increase in the OEE for the original and the proposed configuration. While the increase in quality and performance could be considered not so interesting (at maximum an increase of about $3 \%$ ), the availability could reach an increase of over $40 \%$.

Future research aims to develop the vision system in order to be more effective by studying different methods for image processing and data processing in order to recognize further defects. Moreover, the hoppers could present other configurations that were not considered in the current study. Finally, in future works the presented model will be tested in further case studies. 
Author Contributions: Conceptualization, N.C. and G.R.; Formal analysis, M.F. and R.M.; Investigation, N.C.; Methodology, N.C. and R.M.; Project administration, G.B. and G.R.; Resources, G.B., M.F. and G.R.; Software, N.C.; Supervision, G.B., M.F. and G.R.; Writing—Original Draft, N.C., M.F. and R.M.; Writing—Review \& Editing, G.B., N.C., M.F., R.M. and G.R.

Funding: This research received no external funding.

Conflicts of Interest: The authors declare no conflict of interest.

\section{References}

1. Azzi, A.; Battini, D.; Faccio, M.; Persona, A. Sequencing procedure for balancing the workloads variations in case of mixed model assembly system with multiple secondary feeder lines. Int. J. Product. Res. 2012, 50, 6081-6098. [CrossRef]

2. Barbazza, L.; Faccio, M.; Oscari, F.; Rosati, G. Agility in assembly systems: A comparison model. Assembl. Autom. 2017, 37, 411-421. [CrossRef]

3. Rampersad, H.K. Integrated and Simultaneous Design for Robotic Assembly: Product Development, Planning...; John Wiley \& Sons, Inc.: Hoboken, NJ, USA, 1994.

4. Heilala, J.; Voho, P. Modular reconfigurable flexible final assembly systems. Assembl. Autom. 2001, 21, 20-30. [CrossRef]

5. Battini, D.; Faccio, M.; Persona, A.; Sgarbossa, F. New methodological framework to improve productivity and ergonomics in assembly system design. Int. J. Ind. Ergon. 2011, 41, 30-42. [CrossRef]

6. Cohen, Y.; Faccio, M. The combined effect of multiple models sequencing and absenteeism/turnover on the throughput of assembly lines. In Proceedings of the 17th Industrial Engineering and Management Conference, Tel Aviv, Israel, 27-28 March 2012; pp. 27-28.

7. Edmondson, N.; Redford, A. Generic flexible assembly system design. Assembl. Autom. 2002, 22, 139-152. [CrossRef]

8. Heilala, J.; Montonen, J.; Väätäinen, O. Life cycle and unit-cost analysis for modular reconfigurable flexible light assembly systems. Proc. Inst. Mech. Eng. Part B J. Eng. Manuf. 2008, 222, 1289-1299. [CrossRef]

9. Rosati, G.; Faccio, M.; Carli, A.; Rossi, A. Fully flexible assembly systems (F-FAS): A new concept in flexible automation. Assembl. Autom. 2013, 33, 8-21. [CrossRef]

10. Rosati, G.; Faccio, M.; Barbazza, L.; Rossi, A. Hybrid fexible assembly systems (H-FAS): Bridging the gap between traditional and fully flexible assembly systems. Int. J. Adv. Manuf. Technol. 2015, 81, 1289-1301. [CrossRef]

11. Bevilacqua, M.; Ciarapica, F.; De Sanctis, I.; Mazzuto, G.; Paciarotti, C. A Changeover Time Reduction through an integration of lean practices: a case study from pharmaceutical sector. Assembl. Autom. 2015, 35, 22-34. [CrossRef]

12. "Origin of OEE", OEE Foundation. Available online: http://www.oeefoundation.org/origin-of-oee/ (accessed on 15 July 2015).

13. Cox, D.J.; Lewandowski, W. Design for automation and automated manufacturing of drug testing kits. In Proceedings of the 2005 IEEE International Conference on Systems, Man and Cybernetics, Waikoloa, HI, USA, 12 October 2005; Volume 2, pp. 1194-1199.

14. Engisch, W.E.; Muzzio, F.J. Feedrate deviations caused by hopper refill of loss-in-weight feeders. Powder Technol. 2015, 283, 389-400. [CrossRef]

15. HALCON Solution Guide II-B, Shape-Based Matching. Available online: http://download.mvtec.com/ halcon-9.0-solution-guide-ii-/b-shape-based-matching.pdf (accessed on 4 August 2019).

16. Boothroyd, G. Assembl. Autom. and Product Design, Second Edition (Manufacturing Engineering and Materials Processing); CRC Press, Inc.: Boca Raton, FL, USA, 2005.

17. Zwillinger, D.; Kokoska, S. CRC Standard Probability and Statistics Tables and Formulae; CRC Press, Inc.: Boca Raton, FL, USA, 1999. 